\title{
Post-action memory enhancement: Exploring the temporal relationship between action and memory formation
}

Author names and affiliations

Daisuke Shimane $^{1^{*}}$, Takumi Tanaka ${ }^{2 *}$, Katsumi Watanabe ${ }^{3,4}$, and Kanji Tanaka ${ }^{2}$

* These authors contributed equally to this work.

${ }^{1}$ Department of Psychology, Keio University, Mita, Minato-ku, Tokyo, Japan

${ }^{2}$ Faculty of Arts and Science, Kyushu University, Motooka, Nishi-ku, Fukuoka, Japan

${ }^{3}$ Faculty of Science and Engineering, Waseda University, Shinjuku-ku, Tokyo, Japan

${ }^{4}$ Art \& Design, University of New South Wales, Sydney, Australia

\section{Corresponding author information}

Takumi Tanaka

Faculty of Arts and Science, Kyushu University

Address: Motooka, Nishi-ku, Fukuoka, Japan

Phone number: $+81-92-802-5834$

E-mail: kino31513@artsci.kyushu-u.ac.jp

ORCID ID: 0000-0002-3625-2096 


\begin{abstract}
Actions enhance incidental memory for items that appear in close succession.

However, the role of action processes, such as preparation and execution, on the processes underlying such an interaction is unclear. Here, we examined the temporal dynamics of action-induced memory enhancement. In two experiments, participants performed Go/No-Go tasks while viewing task-unrelated pictures before or after their Go motor responses. Compared to items presented at similar time points in the No-Go trials, items presented after, not before, action execution were consistently better remembered in the subsequent memory tests. Our findings highlight the role of action execution and postaction processes, such as action-effect monitoring, in memory formation.
\end{abstract}

Keywords: memory, action-induced memory enhancement, action-effect monitoring, action planning, attention, attentional boost effect 


\section{Post-action memory enhancement: Exploring the temporal relationship between action and memory formation}

\section{Introduction}

Action selection relies on the unceasing interaction between motor and attentional systems. Humans perform an action in response to external stimuli and monitor the consequence of their action, which, in turn, orients their next action. In driving, for example, one steps on the accelerator after seeing the traffic light turn green and monitors the speed and obstacles coming into sight. Empirical evidence has indicated that attention is guided not only toward to-be-responded stimuli (i.e., targets; Duncan, 1980) but also toward sensory inputs following an action (i.e., action-effect monitoring; Band et al., 2009). After a simple action (e.g., keypress), individuals show spontaneous eye movements toward where an action effect is predicted to appear (Pfeuffer et al., 2016). Such a stimulus captures more visual attention than other external stimuli (Kumar et al., 2015). Furthermore, electroencephalogram (EEG) studies revealed that salient but taskirrelevant action-effects elicit larger amplitudes of event-related potentials associated with involuntary attentional orientation (Nittono \& Ullsperger, 2000; Nittono, 2006). 
Attention also plays a significant role in higher cognitive processes such as memory. People selectively encode what they attend to (Craik et al., 1996). Additionally, increased attention to specific items facilitates the encoding of peripheral information (attentional boost effect, Swallow \& Jiang, 2010); task-irrelevant items are better remembered when presented concurrently with targets than with distractors (Swallow \& Jiang, 2010). At the neural level, target detection is associated with a phasic increase in the release of norepinephrine from the locus coeruleus (Nieuwenhuis et al., 2005). The locus coeruleus-norepinephrine (LC-NE) system is thought to be involved in the attention and has widespread projections to regions responsible for sensory and cognitive processing, including memory (for a review, see Sara, 2009).

Given the known relationships between action and attention and between attention and memory, some researchers began to investigate how action impacts memory and found evidence suggesting that action enhances memory. Chiu and Egner (2015a) showed that people are more likely to remember items that require motor action (Go cue) than those associated with action inhibition (No-Go cue), indicating enhanced memory for behavioral targets. Moreover, even No-Go cues were remembered better when compared with items in the counting task without involving any motor response (Kinder \& Buss, 2020). These findings indicate that merely detecting targets associated with 
action selection or preparing action may induce memory enhancement and that action execution can further facilitate memory formation. To reveal the pure impact of action on memory, Yebra et al. (2019) examined memory for action-unrelated items. In their experiments, participants first performed an encoding Go/No-Go task. The participants executed or withheld a keypress depending on the color of presented frames while also viewing task-irrelevant images presented within the frames (see Fig.1). In the recognition test that followed, participants showed better memory for items presented in the Go trials. Taken together with their evidence from brain imaging and pupillometry measurements, they concluded that taking an action facilitates concurrent memory encoding via recruitment of the LC-NE system.

However, it is still unclear whether action-induced memory enhancement (AIME: memory differences between Go and No-Go trials; Yebra et al., 2019) for unrelated items occurs in processes producing action (i.e., action planning) or processes triggered by action execution. The LC-NE systems are activated in advance of action initiation (Kalwani et al., 2014; Pavlenko \& Kulichenko, 2003; Varazzani et al., 2015). Thus, based on accounts by Yebra et al. (2019), which suggest that action enhances memory by engaging the LC-NE system, encoding can be already facilitated during action planning. On the other hand, the execution of an action may enhance subsequent memory 
encoding by driving attention assignment for action-effect monitoring. We can also assume the existence of temporal dynamics by which these action processes may alternatively or cumulatively influence encoding. That is, in addition to the increased LCNE activation through action planning, action execution might further boost subsequent memory formation.

The potential temporal dynamics of AIME have not yet been sufficiently examined. Yebra et al. (2019) investigated how the mismatch in the stimulus onset from the Go/No-Go cues to unrelated memory items would modulate AIME. In Experiment 2, the items were presented for the first, middle, or last $250 \mathrm{~ms}$ of $750 \mathrm{~ms}$ presentation of response cues in the $0 \mathrm{~ms}, 250 \mathrm{~ms}$, and $500 \mathrm{~ms}$ conditions, respectively (for a similar procedure, see Fig. 1). Based on a non-significant interaction between action requirement and stimulus onsets on memory performance, the authors suggested that a temporal window of AIME is unlikely to exist. However, because the item presentation onsets were determined independently from the participants' actions, they neither assessed memory for items presented immediately before and after actions nor compared them ${ }^{1}$. Moreover,

\footnotetext{
${ }^{1}$ Despite indirect evidence, Yebra et al. (2019) observed a tendency in favor of the potential temporal modulation of action-induced memory enhancement. Two-sided $t$ tests yielded significant memory differences between Go and No-Go items in the 250 $\mathrm{ms}$ and $500 \mathrm{~ms}$ conditions but not in the $0 \mathrm{~ms}$ condition.
} 
given that items were presented during the presentation of Go/No-Go cues in all three conditions, differences between Go and No-Go trials observed there might reflect the effects of target detection as well as action processes ${ }^{2}$. As indicated by the attentional boost effect, the presentation of task-relevant targets improves memory for simultaneously presented items. Such an effect may be more prominent for items presented with action cues, considering that Go/No-Go cues were recognized better than action-unrelated targets (Kinder \& Buss, 2020). Thus, the presentation of Go cues might also enhance memory regardless of action processes.

To specify the process by which encoding facilitation occurs, the current study examined AIME for items presented before and after action execution and directly compared them. If AIME was observed for the items presented before (after) action execution, it would imply that memory encoding was facilitated during action planning (post-action processes). Moreover, when compared to pre-action items, the relative size of AIME for post-action items would be interpreted as the effect of action execution on memory. To separate the impacts of target detection and action execution, we also

${ }^{2}$ Yebra et al. (2019) did not favor the attentional account of AIME by suggesting that the Go cues were equally frequent as the No-Go cues and thus not highly salient. However, in a previous study, the attentional boost effect occurred when targets were as common as distractors (Swallow \& Jiang, 2012). 
controlled for the temporal overlap between Go/No-Go cues and item presentation. The attentional boost effect occurs only when memory items appear during target presentation (Swallow \& Jiang, 2011). Thus, if AIME occurred for items presented after the disappearance of cues, the memory enhancement would not be attributed to target detection. We first conducted an experiment similar to that of Yerba et al. (2019) in which items were presented within response cues before or after actions. In Experiment 2, response cues were briefly presented alone. After that, two items were presented before and after the action execution.

\section{Experiment 1}

\subsection{Methods}

\subsubsection{Participants}

To design an adequate sample size, we used the size of the main effect of action (i.e., Go/No-Go) on memory performance reported in Experiment 2 of Yebra et al. (2019). A priori power analysis using the web-based power application PANGEA ver. 0.2 (https://jakewestfall.shinyapps.io/pangea/) revealed that 32 participants were required to detect a main effect with a similar effect size in a repeated two-way ANOVA $(d=0.566$, $95 \%$ power, $\alpha=.05$ ). Finally, we recruited 36 participants (mean age $=20.69 \pm 1.68$ years; 14 females), considering that some of them would be excluded from the analyses 
because of their inadequate performance. All participants in this study provided written informed consent prior to the experiments.

\subsubsection{Materials}

We used 120 grayscale pictures of icons that depict everyday objects (e.g., books and clocks) or symbols (e.g., hearts and musical notes). We collected the pictures from an open database on the Internet (https://www.dreamstale.com/free-download-220-flatvector-icons/). Half of the pictures were presented in both an encoding task and a recognition task (i.e., 60 old items) and the other half were presented only in the recognition test (i.e., 60 new items). In Experiment 1, the pictures were divided into two datasets and were assigned to old and new items in a counterbalanced way across participants.

\subsubsection{Procedure}

Participants individually performed a modified version of the task developed by Yebra et al. (2019). Our experiment comprised a Go/No-Go task for incidental encoding, an interfering calculation task, and a surprising recognition test (see Fig. 1). In the Go/NoGo task, a colored rectangular frame $(450 \times 300$ pix $)$ and a grayscale picture $(400 \times 250$ pix) appeared simultaneously at the center of a black screen. The frames were presented for $750 \mathrm{~ms}$, with random intervals between 2.3 and 3.3 seconds. The pictures were 
presented simultaneously with (i.e., pre-action condition) or $500 \mathrm{~ms}$ after (i.e., post-action condition) frame presentation onset for $250 \mathrm{~ms}^{3}$. The frames were randomly colored either blue or yellow at equal frequencies. Participants pressed a key when frames with a particular color were presented. Since all pictures assigned to old items were presented at once, participants performed 60 trials of this Go/No-Go task. Next, they engaged in a simple calculation task for one minute. Finally, in the recognition test, frameless pictures comprising old and newly added items are presented. Following the picture presentation for $250 \mathrm{~ms}$, participants indicated whether each picture was presented in the Go/No-Go task ("remember" or "new") with keypresses.

Pictures were randomly ordered in the Go/No-Go task and recognition test. The assignment of old or new items, Go or No-Go items, presentation onset (0 ms or $500 \mathrm{~ms})$, and Go/No-Go-color mapping were counterbalanced across participants. Thirty-two participants were systematically assigned to one of $16(=2 \times 2 \times 2 \times 2)$ patterns for counterbalance. The last four participants were randomly assigned to one of these patterns. The experiment was controlled by PsychoPy3 (Peirce et al., 2019) using a MacMini (Apple).

3 Since the critical contrast was AIME with pre- versus post-actions, we omitted the $250 \mathrm{~ms}$ condition where participants mostly pressed the key during item presentation in Yebra et al.'s Experiment 2. 


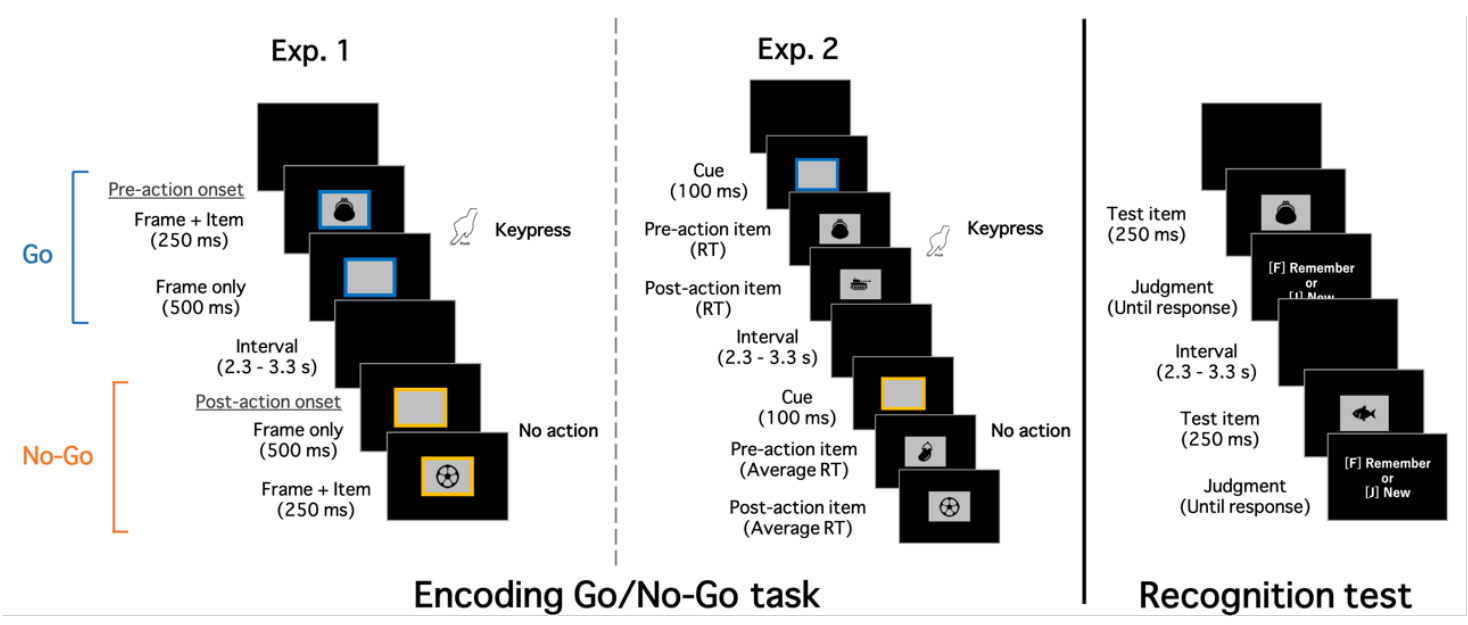

Fig. 1 Schematic illustrations of encoding Go/No-go tasks in Experiments 1 (left) and 2 (middle), and recognition tests in both (right). The procedure of Experiment 1 was similar to that of Experiment 2 in Yebra et al. (2019), except for the absence of the $250 \mathrm{~ms}$ condition. The colored frames were presented for $750 \mathrm{~ms}$, with random intervals varying between 2.3 and 3.3 seconds. The pictures were presented simultaneously with (i.e., preaction condition) or $500 \mathrm{~ms}$ after (i.e., post-action condition) frame presentation onset for $250 \mathrm{~ms}$. The participants pressed a key when frames with a particular color were presented. Next, they engaged in a simple calculation task for one minute. Finally, in the recognition test, frameless pictures comprising old and newly added items were presented. In Experiment 2, participants performed tasks identical to those in Experiment 1, except for the encoding Go/No-Go task in which a colored frame and two items appeared sequentially in each trial. First, a colored frame was presented alone for $100 \mathrm{~ms}$, then a pre-action item was presented. In the Go trials, the pre-action item was presented 
continuously until the participants' keypress responses. It then transformed into a postaction item. Since the post-action item was presented for the RT in that trial, the presentation duration was the same for pre- and post-action items. In the No-Go trials, pre-action items initially appeared for a pre-recorded average RT. They then switched to post-action items for the same amount of time. Next, participants engaged in calculation and recognition tasks similar to those in Experiment 1.

\subsubsection{Statistical Analyses}

In each condition, we calculated the rates of correct "Remember" responses for old items (i.e., hit) and of incorrect "Remember" responses for new items (i.e., false alarm). Similar to Yebra et al. (2019), we used the difference between hit and false alarm rates as a proxy for memory performance. Higher hit rates, compared to false alarms, indicate better memory.

We investigated the impacts of action, presentation onset, and their interaction on memory performance with analysis of variance (ANOVA) and calculated the effect size of partial $\eta^{2}$. Additionally, to assess the occurrence of AIME in each onset condition, we explored the effect size $(d)$ of action comparisons with non-parametric bootstrapped estimation (Halsey et al., 2015; Halsey, 2019). This estimation analysis could supplement significance tests in terms of effect sizes and relative precisions (Claridge-Chang, \& 
Assam, 2016; Ho et al., 2019). We estimated the standardized mean difference of memory performance and 95\% confidence interval (CI) from 5000 bootstrapped samples. Statistical analyses were conducted using $\mathrm{R}$ statistical software (Version 3.1.2; $\mathrm{R}$ Development Core Team, 2020). ANOVAs and calculation of partial $\eta^{2}$ were conducted with the anovakun function (http://riseki.php.xdomain.jp/index.php?FrontPage), and effect sizes of AIME were estimated using the web-based application (https://www.estimationstats.com).

We adopted similar filters for data exclusion by Yebra et al. (2019). We did not analyze data from four participants who failed to press the key in Go trials (more than $10 \%$ of trials) and one participant whose memory performance in the recognition test was less than zero (i.e., chance level). If participants made an incorrect response in the Go/NoGo task, the items showed in those trials were excluded from the analyses. Importantly, since the critical contrast was AIME with pre- versus post-actions, we finally excluded the data from analyses when response times (RTs) were shorter than $250 \mathrm{~ms}$, or longer than $500 \mathrm{~ms}$ ( $\sim 19 \%$ of all Go trials). This filter was not adopted by Yebra et al. (2019). 
(a)

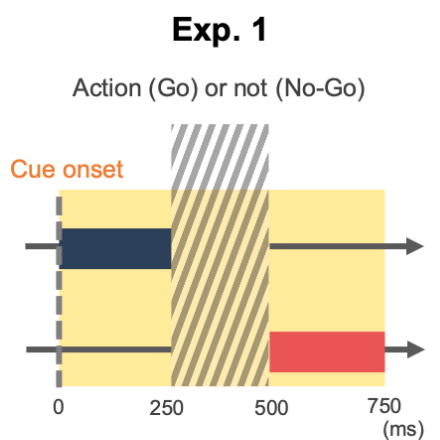

(b)

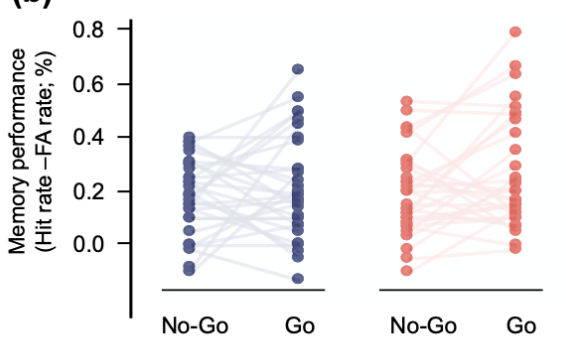

(c)

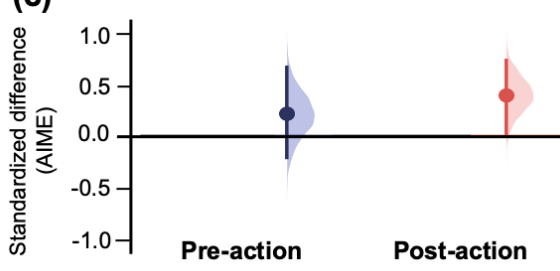

Exp. 2
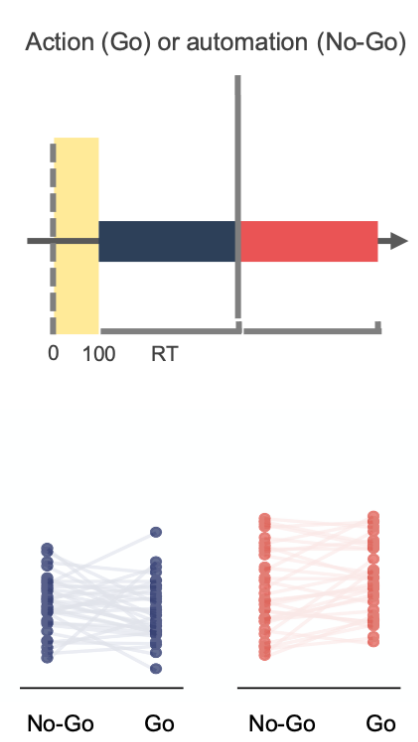

Overall

(Exp. 1 \& Exp. 2)

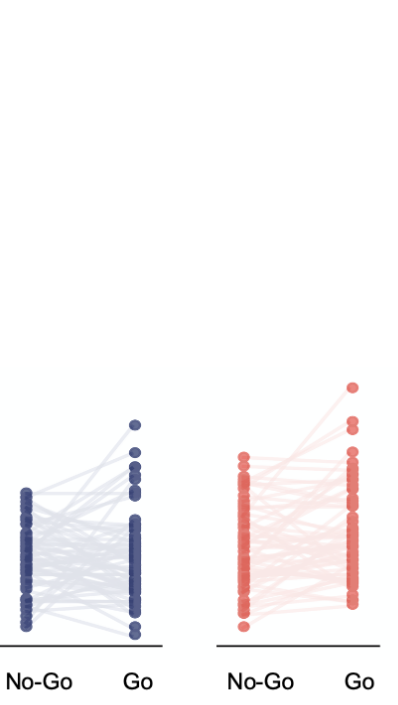

Fig. 2 The effects of actions on memory performance as a function of the temporal relationship between actions and item presentation in each experiment. Schemas indicating event timelines in each experiment are shown in (a). Navy blue (Red) rectangles represent item presentation before actions (after actions). In Experiment 1, the participants pressed the keys at arbitrary times. Therefore, the shaded field in the timeline represents the range of possible reaction times. The gray line in the timeline of Experiment 2 represents the specific response times. Participants' memory performance is plotted in (b) for both the No-Go and Go items. Each estimated standard mean 
difference (i.e., effect size of AIME) is represented by dots and plotted in (c) with a bootstrap sampling distribution. The vertical error bars depict $95 \%$ confidence intervals.

\subsection{Results and Discussion}

A 2 (action: Go vs. No-Go) $\times 2$ (onset: pre- vs. post-action) within-participants ANOVA was conducted (Fig. 2). The results showed neither a significant main effect of action $\left(F_{1,30}=3.219, p=.083\right.$, partial $\left.\eta^{2}=0.097\right)$, onset $\left(F_{1,30}=1.249, p=.273\right.$, partial $\eta^{2}=$ $0.040)$, nor interaction $\left(F_{1,30}=0.852, p=.363\right.$, partial $\left.\eta^{2}=0.028\right)$. The $t$-tests and effect size estimation to assess AIME confirmed the better memory for Go items in the postaction condition $\left(t_{30}=2.085, p=.046 ; d[95 \%\right.$ CI $\left.0.0161,0.729]=0.389\right)$, but not in the pre-action condition $\left(t_{30}=0.937, p=.357 ; d[95 \%\right.$ CI $\left.-0.216,0.662]=0.21\right)$.

The results of Experiment 1 were inconclusive. While a more robust AIME after actions was indicated, we failed to observe a significant interaction effect in ANOVA. Furthermore, our experiment did not replicate the significant main effect of action observed in Yebra et al. (2019). In pre-registered Experiment 2, to investigate the temporal dynamics in AIME more directly, we presented pre-action items and post-action items before and after keypresses in the identical Go trials. In other words, the items were switched by actions. Moreover, to rule out the influence of target detection (i.e., 
attentional boost effect), we temporally separated item presentation from the Go/No-Go cues.

\section{Experiment 2}

\subsection{Methods}

\subsubsection{Participants}

Since the effect of temporal relationship between actions and item presentation on memory has not been assessed directly, there was no specific standard for the effect size. Thus, assuming that the target interaction effect would have a low-medium effect size, we calculated the adequate sample size in a repeated $2 \times 2$ two-way ANOVA $(d=0.40$, $95 \%$ power, $\alpha=.05$ ). This priori power analysis indicated that $32 \mathrm{had}$ an adequate sample size. We recruited 36 new participants (mean age $=21.11 \pm 1.98$ years; 17 females), similar to Experiment 1, considering that some participants would be excluded from the analyses due to their inadequate performance.

\subsubsection{Materials}

We used newly collected 200 grayscale pictures of icons from an open database on the Internet (https://icooon-mono.com). The pictures were divided into two predetermined datasets, which were assigned to old and new items in a counterbalanced way across participants (i.e., 100 old items and 100 new items). 


\subsubsection{Procedure}

Before the encoding task, participants performed 60 trials (i.e., 30 Go and 30 NoGo trials) of a Go/No-Go task without item presentations. The same blue and yellow frames were presented for $100 \mathrm{~ms}$ in random order with random 2.3-3.3 intervals. Participants pressed a key in response to frames with Go color and restrained response to frames with No-Go color. In the Go trials, the participants' RTs were recorded.

Then, participants performed identical tasks to Experiment 1, except for the encoding Go/No-Go task, in which two items appeared sequentially in each trial after the presentation of frames. First, a Go (No-Go) frame was presented for $100 \mathrm{~ms}$, and then a pre-action item was presented. In Go trials, the pre-action item was presented until participants' keypresses. It then transformed into a post-action item. Since the post-action item was presented for the RT in that trial, the presentation duration was the same for preand post-action items. In No-Go trials, pre-action items initially appeared for a prerecorded average RT. They then switched to post-action items for the same amount of time. RTs were constantly recorded in Go trials during the encoding task. The average RT was updated for each Go trial. Next, participants engaged in similar calculation and recognition tasks as in Experiment 1. 
In Experiment 2, the items were randomly assigned to Go/No-Go and pre/postaction conditions. The assignment of items to old/new items and colors of the frame was counterbalanced across participants. This experiment was controlled by MATLAB (The Math Works, Natick, MA) with Psychtoolbox (Brainard, 1997; Kleiner et al., 2007; Pelli, 1997) using a MacMini (Apple).

\subsubsection{Statistical Analyses}

The measures and analysis procedures were the same as those in Experiment 1. One participant who failed to press the key in Go trials (more than $10 \%$ of trials) in the encoding task and two whose memory performance in the recognition test was less than zero (i.e., chance level) were excluded from the analysis. Items showed in trials with incorrect Go/No-Go responses were excluded from the analyses.

\subsection{Results and Discussion}

A 2 (action: Go vs. No-Go) $\times 2$ (onset: pre- vs. post-action) within-participants ANOVA revealed a significant interaction $\left(F_{1,32}=6.096, p=.019\right.$, partial $\left.\eta^{2}=0.160\right)$. Analyses with simple main effects indicated that actions improved memory for postaction items $\left(F_{1,32}=4.764, p=.037\right.$, partial $\left.\eta^{2}=0.130\right)$, but not for pre-action items $\left(F_{1,32}=\right.$ $1.338, p=.256$, partial $\left.\eta^{2}=0.040\right)$. Moreover, the main effect of onset was significant $\left(F_{1,32}=22.975, p<.001\right.$, partial $\left.\eta^{2}=0.418\right)$, revealing better memory for post-action items 
than pre-action items. The main effect of action was not significant $\left(F_{1,32}=0.217, p=.645\right.$, partial $\left.\eta^{2}=0.007\right)$

Again, $t$-tests and estimations (see Fig. 2) confirmed reliable memory enhancement for post-action items $\left(t_{32}=2.183, p=.037 ; d[95 \%\right.$ CI $\left.0.045,0.547]=0.273\right)$, but not for pre-action items $\left(t_{32}=1.157, p=.256 ; d[95 \%\right.$ CI $\left.-0.646,0.213]=-0.241\right)$. Such a contrast was also observed when testing pooled data of Experiments 1 and 2 (preaction items: $t_{32}=1.157, p=.256 ; d[95 \%$ CI $-0.646,0.213]=-0.241$, post-action items: $t_{32}=2.183, p=.037 ; d[95 \%$ CI $0.045,0.547]=0.273$; see Fig. 2).

\section{General Discussion}

Our objective was to investigate whether memory enhancement occurs in the processes producing action and/or processes triggered by action execution. In the two experiments, we compared memory enhancement for items presented before versus after actions. Compared to items presented at similar time points in the No-Go trials, items presented after, not before, action execution were consistently better remembered in the subsequent memory tests. In Experiment 2, we directly confirmed that the memory difference between the Go and No-Go items was significantly larger for post-action items than pre-action items. These data indicate that actions facilitate encoding processes after their execution rather than during action planning. 
The impact of action execution on subsequent memory might not have been clearly observed so far due to the co-occurrence of the attentional boost effect. Experiment 1 did not yield strong evidence for the presence of AIME or its temporal dynamics. Since all items were presented simultaneously with the response cues, the presentation of Go cues might have elicited an increased attention and enhanced memory for both pre- and post-action items. Moreover, it is also possible that even No-Go cues facilitated attention and thereby encoding, reducing AIME. These possibilities concur with previous studies, suggesting that the memory difference between Go and No-Go items is subtle (Chiu \& Egner, 2015a, 2015b; Kinder \& Buss, 2020) and sometimes not reliable (Kinder \& Buss, 2020; Yebra et al., 2019). In these studies, memory items themselves served as response cues (Chiu \& Egner, 2015a, 2015b; Kinder \& Buss, 2020) or were presented concurrently with response cues (Yebra et al., 2019). To assess the pure impact of action execution on memory, it seems important to control for both the temporal relationship between actions and item presentation and that between response cue and item presentation. In this sense, Experiment 2 provided new evidence for action-induced memory enhancement for the items that were temporally separated from response cues.

A potential explanation for the observed post-action memory enhancement is that action execution might induce additional assignment of attentional resources. This 
interpretation is in line with the framework of the action-effect monitoring, that is, people are sensitive to sensory inputs following their actions, regardless of whether they are relevant to the task (Band et al., 2009). Nevertheless, the causal relationship between memory enhancement and attention or the activation of the LC-NE system should be further investigated. It is still unclear whether the activation of the LC-NE system during action planning and memory enhancement after action execution are independent of each other. Given that LC-NE systems are involved in attentional function (Sara, 2009), the increased LC-NE activity may contribute to subsequent attentional boost. Alternatively, if the norepinephrine release itself enhances memory encoding, as suggested by Yebra et al. (2019), the cumulative activation of the LC-NE system through action processes might result in robust memory enhancement. Future studies can address this hypothesis by investigating the neural mechanisms underlying temporal dynamics using high temporal resolution measures such as EEG.

Another explanation for our findings is that action execution may facilitate memory for subsequent stimuli via higher cognitive processes other than attention. The literature has shown that people can perceive a sensory event following their actions as their action effects, regardless of objective contingency (e.g., Yon et al., 2020). The (illusory) self-generation may be accompanied by unique subjective experiences such as 
the sense of agency or ownership over the items (Gallagher, 2000). This agency (Hon \& Yeo, 2021) or self-relevance (Kim \& Johnson, 2012) over items results in better incidental memory. At this point, we could not elucidate whether these subjective experiences would contribute to encoding. Future research can quantify them with explicit (Hon \& Yeo, 2021) or implicit (e.g., intentional binding, Haggard et al. 2003) measures and assess their relationship with memory performance. Post-action memory enhancement may also be related to the benefits of active learning. For example, self-directed learning, such as choosing the items to study (Kornell \& Metcalfe, 2006) and deciding the order and timing of study (Markant et al., 2014), enhance performance in subsequent memory tests. In this line of work, participants realize the presence of a later test and intentionally memorize the items. Thus, they may use encoding strategies that can potentially improve their performance; for example, spending more time and cognitive effort for the difficult-toencode items (Tullis \& Benjamin, 2011). In contrast, the participants in our experiments could not adopt such explicit strategies due to the incidental memory test. Therefore, although the post-action memory enhancement revealed here may contribute to some forms of active learning, it likely has a different source from the known advantages of explicit mnemonics. 
The current study is the first to empirically demonstrate a unique relationship between action execution and memory; action execution improves memory for subsequent stimuli. Our data are unlikely explained by known attentional boost to behavioral target but implicate potential involvement of action-effect monitoring. Such processes may help us learn the consequences of our own actions. This idea is consistent with another aspect of the LC-NE system that is involved in detecting and learning from negative or unexpected action effects (Clewett et al., 2014). While there is a large volume of studies on memory for actions (i.e., what one did) and cues of action (i.e., what led the action), little is known about the memory processes of action effects (i.e., what one caused). Considering that memory for action effects plays a crucial role in guiding action selection and composing the narrative self, our findings provide a stepping stone toward further work in this fruitful area. 


\section{Declarations of interest:}

The authors declare that they have no conflict of interest.

\section{Funding:}

This work was supported by Grants-in-Aid by Nakayama Hayao Foundation for

Science, Technology and Culture, Japan (R1-B-56) and JSPS KAKENHI (JP20K22269)

to TT, JSPS KAKENHI (JP18H03505) to KT, and JSPS KAKENHI (JP17H00753 and JP17H06344) and CREST (JPMJCR14E4) to KW.

\section{Acknowledgements:}

We would like to thank Editage (www.editage.com) for English language editing.

Ethics: This study was approved by the institutional review board of Waseda University (2015-033) and conducted in accordance with the ethical standards of the 1964 Declaration of Helsinki. 
Data Availability: The datasets analyzed during the current study are available in the Open Science Framework repository at: https://osf.io/9gbxq/?view_only=a3d21d84c3a24199b487d5569e271cdc

Code availability: The Python sources for analyses during the current study are available in the Open Science Framework repository at: https://osf.io/9gbxq/?view_only=a3d21d84c3a24199b487d5569e271cdc

Pre-registration: The pre-registered design and analyses of Experiment 2 can be found in the Open Science Framework repository at:

https://osf.io/9gbxq/?view_only=a3d21d84c3a24199b487d5569e271cdc 


\section{References}

Band, G.P.H., van Steenbergen, H., Ridderinkhof, K.R., Falkenstein, M., \& Hommel, B. (2009). Action-effect negativity: Irrelevant action effects are monitored like relevant feedback. Biological Psychology, 82(3), 211-218.

Brainard, D. H. (1997). The psychophysics toolbox. Spatial Vision, 10, 443-446.

Chiu, Y.-C., \& Egner, T. (2015a). Inhibition-induced forgetting: when more control leads to less memory. Psychological Science, 26(1), 27-38. https://doi.org/10.1177/0956797614553945

Chiu, Y. C., \& Egner, T. (2015b). Inhibition-induced forgetting results from resource competition between response inhibition and memory encoding processes. Journal of Neuroscience, 35, 11936-11945.

Claridge-Chang, A., \& Assam, P. N. (2016). Estimation statistics should replace significance testing. Nature Methods, 13(2), 108-109. https://doi.org/10.1038/nmeth.3729

Clewett, D., Schoeke, A., \& Mather, M. (2014). Locus coeruleus neuromodulation of memories encoded during negative or unexpected action outcomes. Neurobiology of Learning and Memory, 111, 65-70. 
Craik, F. I., Govoni, R., Naveh-Benjamin, M., \& Anderson, N. D. (1996). The effects of divided attention on encoding and retrieval processes in human memory. Journal of Experimental Psychology: General, 125(2), 159-180.

Duncan, J. (1980). The locus of interference in the perception of simultaneous stimuli. Psychological Review, 87(3), 272.

Gallagher, S. (2000). Philosophical conceptions of the self: implications for cognitive science. Trends in Cognitive Sciences, 4(1), 14-21.

Haggard, P., Clark, S., \& Kalogeras, J. (2002). Voluntary action and conscious awareness. Nature neuroscience, 5(4), 382-385.

Halsey, L. G., Douglas C. E., Sarah L. V., \& Gordon B. D. (2015). The fickle p value generates irreproducible results. Nature Methods, 12(3), 179-185.

Halsey, L. G. (2019). The reign of the p-value is over: what alternative analyses could we employ to fill the power vacuum?. Biology Letters, 15(5), 20190174.

Ho, J., Tumkaya, T., Aryal, S., Choi, H., \& Claridge-Chang, A. (2019). Moving beyond p values: data analysis with estimation graphics. Nature Methods, 16(7), 565-566.

Hon, N., \& Yeo, N. Having a sense of agency can improve memory. Psychonomic Bulletin \& Review, 1-7. 
Kalwani, R. M., Joshi, S., \& Gold, J. I. (2014). Phasic activation of individual neurons in the locus ceruleus/subceruleus complex of monkeys reflects rewarded decisions to go but not stop. Journal of Neuroscience, 34(41), 13656-13669.

Kim, K., \& Johnson, M. K. (2012). Extended self: medial prefrontal activity during transient association of self and objects. Social Cognitive and Affective Neuroscience, 7(2), 199-207.

Kinder, K. T., \& Buss, A. T. (2020). The effect of motor engagement on memory: Testing a motor-induced encoding account. Memory \& Cognition, 1-14. https://doi.org/10.3758/s13421-020-01113-6

Kleiner, M., Brainard, D., Pelli, D., Ingling, A., Murray, R., \& Broussard, C. (2007). What's new in Psychtoolbox-3. Perception, 36(14), 1-16.

Kornell, N., \& Metcalfe, J. (2006). Study efficacy and the region of proximal learning framework. Journal of Experimental Psychology: Learning, Memory, and Cognition, 32(3), 609-622.

Kumar, N., Manjaly, J.A., \& Sunny, M.M. (2015). The relationship between action-effect monitoring and attention capture. Journal of Experimental Psychology: General, 144(1), 18-23. 
Markant, D., DuBrow, S., Davachi, L., \& Gureckis, T. M. (2014). Deconstructing the effect of self-directed study on episodic memory. Memory \& Cognition, 42(8), $1211-1224$.

Nieuwenhuis, S., Aston-Jones, G., \& Cohen, J. D. (2005). Decision making, the P3, and the locus coeruleus-norepinephrine system. Psychological Bulletin, 131(4), 510.

Nittono, H., \& Ullsperger, P. (2000). Event-related potentials in a self-paced novelty oddball task. NeuroReport, 11(9), 1861-1864.

Nittono, H. (2006). Voluntary stimulus production enhances deviance processing in the brain. International Journal of Psychophysiology, 59(1), 15-21.

Pavlenko, V. B., \& Kulichenko, A. M. (2003). Self-initiated motor behavioral act-related neuronal activity in the cat locus coeruleus. Neurophysiology, 35(1), 29-37.

Peirce, J., Gray, J. R., Simpson, S., MacAskill, M., Höchenberger, R., Sogo, H., ... \& Lindeløv, J. K. (2019). PsychoPy2: Experiments in behavior made easy. Behavior Research Methods, 51(1), 195-203.

Pfeuffer, C.U., Kiesel, A., \& Huestegge, L. (2016). A look into the future: Spontaneous anticipatory saccades reflect processes of anticipatory action control. Journal of Experimental Psychol: General, 145(11), 1530-1547. 
Pelli, D. G. (1997). The VideoToolbox software for visual psychophysics: Transforming numbers into movies. Spatial Vision. 10(4), 437-442.

R Core Team. (2020). R: A language and environment for statistical computing. R Foundation for Statistical Computing, Vienna, Austria. URL https://www.Rproject.org/.

Sara, S. J. (2009). The locus coeruleus and noradrenergic modulation of cognition. Nature Reviews Neuroscience. 10(3), 211-223.

Swallow, K. M., \& Jiang, Y. V. (2010). The Attentional Boost Effect: Transient increases in attention to one task enhance performance in a second task. Cognition, 115(1), $118-132$.

Swallow, K. M., \& Jiang, Y. V. (2011). The role of timing in the attentional boost effect. Attention, Perception, \& Psychophysics, 73(2), 389-404.

Swallow, K. M., \& Jiang, Y. V. (2012). Goal-relevant events need not be rare to boost memory for concurrent images. Attention, Perception \& Psychophysics, 74(1), 7082.

Tullis, J. G., \& Benjamin, A. S. (2011). On the effectiveness of self-paced learning. Journal of Memory and Language, 64(2), 109-118. 
Varazzani, C., San-Galli, A., Gilardeau, S., \& Bouret, S. (2015). Noradrenaline and dopamine neurons in the reward/effort trade-off: a direct electrophysiological comparison in behaving monkeys. Journal of Neuroscience, 35(20), 7866-7877. https://doi.org/10.1523/JNEUROSCI.0454-15.2015

Yebra, M., Galarza-Vallejo, A., Soto-Leon, V., Gonzalez-Rosa, J. J., de Berker, A. O., Bestmann, S., Oliviero, A., Kroes, M. C. W., \& Strange, B. A. (2019). Action boosts episodic memory encoding in humans via engagement of a noradrenergic system. Nature Communications, 10(1), 3534. https://doi.org/10.1038/s41467-019-113588

Yon, D., Bunce, C., \& Press, C. (2020). Illusions of control without delusions of grandeur. Cognition, 205, 104429. 